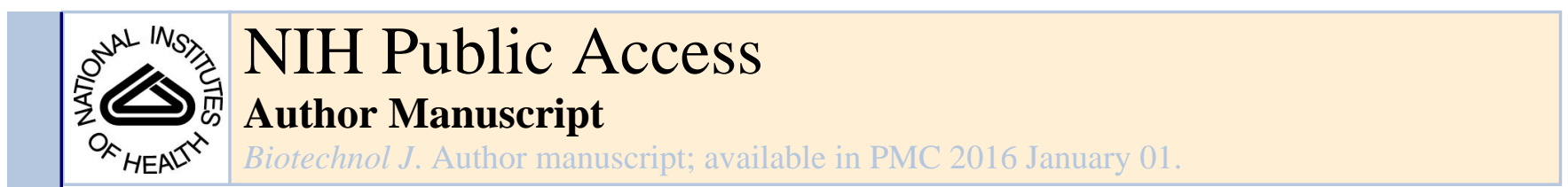

Published in final edited form as:

Biotechnol J. 2015 January ; 10(1): 199-209. doi:10.1002/biot.201400354.

\title{
Glycoarrays with engineered phages displaying structurally diverse oligosaccharides enable high-throughput detection of glycan-protein interactions
}

\author{
Eda Çelik ${ }^{1,2,3}$, Anne A. Ollis ${ }^{1}$, Yi Lasanajak ${ }^{4}$, Adam C. Fisher ${ }^{5}$, Göksu Gür ${ }^{3}$, David F. \\ Smith $^{4}$, and Matthew P. DeLisa ${ }^{1, *}$ \\ ${ }^{1}$ School of Chemical and Biomolecular Engineering, Cornell University, Ithaca, NY 14853 USA \\ 2Department of Chemical Engineering, Hacettepe University, Beytepe, 06800 Ankara, Turkey \\ ${ }^{3}$ Institute of Science, Bioengineering Division, Hacettepe University, Beytepe, 06800 Ankara, \\ Turkey \\ ${ }^{4}$ National Center for Functional Glycomics, Department of Biochemistry, Emory University School \\ of Medicine, Atlanta, GA 30322 USA
}

${ }^{5}$ Glycobia, Inc, 410 Weill Hall, Ithaca, NY 14853

\section{Abstract}

Glycan microarrays have become a powerful platform to investigate the interactions of carbohydrates with a variety of biomolecules. However, the number and diversity of glycans available for use in such arrays represents a key bottleneck in glycan array fabrication. To address this challenge, we describe a novel glycan array platform based on surface patterning of engineered glycophages that display unique carbohydrate epitopes. Specifically, we show that glycophages are compatible with surface immobilization procedures and that phage-displayed oligosaccharides retain the ability to be recognized by different glycan-binding proteins (e.g., antibodies, lectins) after immobilization. A key advantage of glycophage arrays is that large quantities of glycophages can be produced biosynthetically from recombinant bacteria and isolated directly from bacterial supernatants without laborious purification steps. Taken together, the glycophage array technology described here should help to expand the diversity of glycan libraries and provide a complement to the existing toolkit for high-throughput analysis of glycan-protein interactions.

\section{Keywords}

filamentous phage; functional glycomics; glycan-binding protein; glycoarray; lectin

\footnotetext{
*To whom correspondence should be addressed: Matthew P. DeLisa, Phone: 607-254-8560; Fax: 607-255-9166; md255@ cornell.edu. Conflict of Interest Statement

The authors declare no commercial or financial conflict of interest
} 


\section{Introduction}

Carbohydrate chains known as glycans are involved in myriad molecular interactions and are crucial for the development, growth, function, and survival of many organisms [1]. Their importance is derived in part from the incredible diversity of forms that have been observed in nature, either as free structures or as conjugates to other biomolecules (such as proteins, lipids, and nucleic acids). In fact, the spectrum of all glycan structures, known as the glycome, in some organisms is so large that it exceeds the number of proteins encoded in the genome by orders of magnitude [2].

To date, substantial knowledge regarding glycans has been gained from studies employing traditional analytical tools. However, there remains a pressing need for high-throughput techniques that allow systematic investigation of structurally and functionally important glycans, which often involve glycan-binding proteins (GBPs) that recognize specific glycan sequences and thereby 'decode' the complex structural information in glycans. A major technical breakthrough along these lines was the development of printed glycan microarrays, in which many defined glycan structures are immobilized on a solid support in a spatially defined arrangement and simultaneously presented to GBPs or microorganisms including viruses and bacteria (reviewed in [3-8]). Such microarrays enable multiplex analysis of glycan-protein interactions using only tiny amounts of material and thus have emerged as a powerful methodology for decoding the informational content of the glycome. One of the first reports of such a system was in 2002 by Wang et al. who described the fabrication of a carbohydrate array to identify specific microbial polysaccharides that interacted with human antibodies [9]. Since that time, numerous reports have demonstrated the use of glycan microarrays for determining the glycan-binding specificity of GBPs and shedding light on key mechanistic details underlying mammalian biology, host-pathogen interactions, and immune recognition of glycans $[3,5,10,11]$.

In general, the glycan substrates for most microarrays are either synthesized via chemical, enzymatic and/or chemo-enzymatic routes, which can be expensive; or isolated from natural sources such as cells, tissues, pathogens, milk or urine, which yields low amounts of glycan and requires several purification steps [4, 7]. As a result, most glycan microarray studies only use a small fraction of the total structural diversity found in nature. While this has been sufficient to obtain useful results in a variety of applications $[9,12-16]$, the full potential of defined glycan microarrays will only be realized by (i) increasing the quantity and diversity of glycan structures and (ii) developing reliable and reproducible chemistries for the immobilization of chemically and structurally diverse glycan probes onto a solid support with retention of functionality. Hence, the chemistries related to the synthesis, harvesting, and immobilization of glycans are paramount for microarray technology development.

Here, we describe a facile alternative for glycan array fabrication using engineered phages that display defined glycan epitopes, so-called "glycophages" $[17,18]$. Since its inception in 1985 [19], phage display has been used primarily for library-based selection of ligandbinding proteins [20-23] as well as stabilized protein variants, regulatable enzymes and protein catalysts [23-25]. While far less common, recombinant phages can also be immobilized on a solid support in a spatially defined arrangement such that phage-displayed 
proteins or peptides are accessible for subsequent high-throughput protein-protein interaction screening. For example, microarrays have been patterned with recombinant phages displaying antibody clones for the detection of leukocyte surface antigens [26], small peptides for the diagnosis of the immune response in patients with cancer [27] or HIV [28], and even cDNA libraries for the diagnosis of non-small cell lung cancer [29]. In all of these cases, phage particles were entirely compatible with the microarray patterning process and the displayed molecules (e.g., proteins, peptides) were accessible for subsequent proteinprotein interaction analysis using standard microarray scanners. Building on these ideas, we have combined the immobilization compatibility of phage particles with our recent demonstration of glycan display on filamentous phage [17] to create a novel glycan array technology for detecting GBP interactions. Specifically, we show that glycophages are compatible with surface immobilization procedures, can be modified with different glycan structures, and retain the ability following immobilization to be recognized by different GBPs (e.g., antibodies, lectins) that recognize the displayed oligosaccharides. The advantages of glycophage-based arrays include the low cost and scalability of phage/glycan production, which are biosynthetic processes involving the cultivation of recombinant Escherichia coli cells, and the ease with which glycophages can be recovered from the bacterial supernatant without laborious purification steps. For these reasons, we anticipate that glycophage arrays will become a useful tool for identifying and profiling GBPs, including mammalian lectins (C-type lectins, galectins, and siglecs), plant lectins, antibodies, viral and bacterial lectins, and intact viruses.

\section{Materials and Methods}

\section{Bacterial strains and growth conditions}

E. coli strain DH5a ( $\mathrm{F}^{-}$endA1 glnV44 thi-1 recAl relA1 gyrA96 deoR nupG $\varphi 80$ lacZ $\Delta \mathrm{M} 15$ $\Delta($ lacZYA- $\left.\arg F) \mathrm{U} 169, h s d R 17\left(\mathrm{r}_{\mathrm{K}}{ }^{-} \mathrm{m}_{\mathrm{K}}{ }^{+}\right), \lambda-\right)$ was used for cloning of phagemids, while strain TG1 (supE hsd $\Delta 5$ thi $\Delta$ (lac-proAB) F' [traD36 proABlacIlacZ $\Delta M 15$ ] was used for phage titering. To quantify phage titers, fresh TG1 cells were infected with the recovered phages and then selected on ampicillin to determine colony-forming units (CFUs). Strain TG1 lacking the waaL gene (TG1 $\Delta w a a L)$ was generated by P1vir phage transduction using strain TG1 as recipient and strain BW25113 waaL::Kan from the Keio collection [30] as donor. The resulting strain was used for all glycophage production. VCSM13 (Stratagene) was used as the helper phage and propagated in wild-type TG1 cells as described [31]. E. coli strains were grown in Luria-Bertani (LB) or 2xTY medium (during phage production) at $37^{\circ} \mathrm{C}$ or $30^{\circ} \mathrm{C}$ (induction phase). Culture medium was supplemented with $1 \%$ glucose (w/v) or $0.2 \%$ (w/v) L-arabinose (induction phase), as well as with the appropriate antibiotics at the following concentrations: $100 \mu \mathrm{g} / \mathrm{ml}$ ampicillin (Amp), $20 \mu \mathrm{g} / \mathrm{ml}$ chloroamphenicol $(\mathrm{Cm})$, and $35 \mu \mathrm{g} / \mathrm{ml}$ kanamycin (Kan). M9 minimal medium was used to select for the presence of the F' plasmid when needed.

\section{Construction of phagemids}

The phagemids constructed here were based on the phagemid pBAD-MBP ${ }^{D^{2 N A T}}$-g3p [17], encoding E. coli maltose-binding protein (MBP) modified with a C-terminal DQNAT motif, and cloned using standard protocols. To generate phagemid pBAD-MBP ${ }^{4 D_{Q}}{ }_{-}{ }_{-} 3 \mathrm{p}$, 
DNA encoding MBP $4 x$ DQNAT was PCR-amplified from plasmid pTrc-MBP-GT [32] as template. Next, pBAD-MBP ${ }^{D^{2 N A T}}$-g3p was digested with EcoRI-Sall to remove $\mathrm{MBP}^{\mathrm{DQNAT}}$, followed by ligation of PCR-amplified MBP ${ }^{4 \mathrm{DQNAT}}$ into the same sites resulting in $\mathrm{pBAD}-\mathrm{MBP}^{4 \times D Q N A T}-\mathrm{g} 3 \mathrm{p}$. To generate the phagemid $\mathrm{pBAD}-\mathrm{MBP}^{4 \times D Q N A T}$ $\mathrm{CT}:$ PglB for bicistronic expression of $\mathrm{MBP}^{4 \times D Q N A T}$ fused to the $\mathrm{C}$-terminal domain of $\mathrm{g} 3 \mathrm{p}$ (CT; residues 230-406) [33] and PglB, the bicistronic phagemid pBAD-AcrA::PglB (laboratory stock) was digested with EcoRI and $X m a$ I to remove the gene encoding AcrA. Next, DNA encoding MBP ${ }^{4 D Q N A T}$-g3p was PCR-amplified from $p B A D-M B P^{4 x D Q N A T}$ g3p as template and then ligated into EcoRI-XmaI digested pBAD-AcrA::PglB, resulting in phagemid pBAD-MBP ${ }^{4 x D Q N A T}$-g3p::PglB. The gene encoding full-length g3p (406 amino acids) was removed from $\mathrm{pBAD}-\mathrm{MBP}^{4 \times D Q N A T-} \mathrm{g} 3 \mathrm{p}:$ :PglB by digestion with SalI and $\mathrm{XmaI}$ and DNA encoding the C-terminal domain of $\mathrm{g} 3 \mathrm{p}$ (residues 230-406) [33] was ligated into

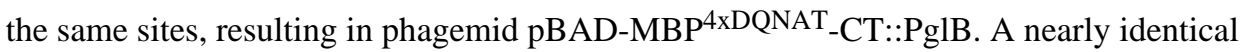
strategy was used to construct the negative control phagemids $\mathrm{PBAD}-\mathrm{MBP}^{4 \times A Q N A T}$. $\mathrm{g} 3 \mathrm{p}:: \mathrm{PglB}$ and $\mathrm{pBAD}-\mathrm{MBP}^{4 \mathrm{xAQNAT}} \mathrm{CT}:: \mathrm{PglB}$ in which all four acceptor motifs were changed to AQNAT, a sequence that is inefficiently recognized by Campylobacter jejuni PglB $[34,35]$. All phagemids were confirmed by DNA sequencing.

Plasmids used for glycan biosynthesis included: pMW07pgl $\Delta \mathrm{B}$ for the $C$. jejuni heptasaccharide glycan (Cj-hepta) [35]; pACYCpgl4 for the C. lari hexasaccharide glycan (Cl-hexa) [36]; pYCG for the eukaryotic $\mathrm{Man}_{3} \mathrm{GlcNAc}_{2}$ core glycan (Man3) [37]; pMW07O78 for E. coli O78 O-antigen (Ec-OAg (O78); laboratory stock); pMW07-O148 for E. coli O148 O-antigen (Ec-OAg (O148); laboratory stock); pGAB2 for Francisella tularensis SchuS4 O-antigen (Ft-OAg) [38], pLPS2 for Psuedomonas aeruginosa O11 O-antigen (PaOAg) [39], and pSS37 for Shigella dysenteriae serotype 1 O-antigen (Sd-OAg) [40].

\section{Production and purification of glycophage particles}

A single colony of $E$. coli TG1 $\Delta$ waaL carrying a phagemid, either pBAD-MBP ${ }^{4 x D Q N A T}$ $\mathrm{g} 3 \mathrm{p}:: \mathrm{PglB}$ or $\mathrm{pBAD}-\mathrm{MBP}^{4 \times D Q N A T} \mathrm{CT}:: \mathrm{PglB}$, and a plasmid for glycan biosynthesis was inoculated into $6 \mathrm{~mL}$ of $\mathrm{M} 9$ minimal medium supplemented with Amp and $\mathrm{Cm}$ and shaken at $37^{\circ} \mathrm{C}$ overnight. The following day, cells were subcultured into $40 \mathrm{~mL}$ of $2 x T Y$ medium supplemented with $\mathrm{Amp}, \mathrm{Cm}$, and glucose such that the initial $\mathrm{OD}_{600}$ was 0.06 and shaken at $37^{\circ} \mathrm{C}$. The culture was superinfected with $3.2 \times 10^{11} \mathrm{CFUs}$ of VCSM13 helper phage when the $\mathrm{OD}_{600}$ reached 0.5-0.6 and incubated for $30 \mathrm{~min}$ at $37^{\circ} \mathrm{C}$ without shaking. For recovering cells, flasks were incubated for $10 \mathrm{~min}$ at $37^{\circ} \mathrm{C}$ with shaking and then centrifuged $\left(3,300 \times g, 10 \mathrm{~min}, 16^{\circ} \mathrm{C}\right)$. The resulting cell pellet was resuspended in $40 \mathrm{~mL} 2 \mathrm{xTY}$ medium supplemented with Amp, Cm and Kan. Resuspended cells were cultured for $30 \mathrm{~min}$ at $30^{\circ} \mathrm{C}$ with shaking, after which protein expression was induced with $0.2 \%$ arabinose for $16 \mathrm{~h}$. For glycophage recovery, the culture supernatant was separated from the cells by centrifugation $\left(6,000 \times g, 15 \mathrm{~min}, 4^{\circ} \mathrm{C}\right)$. Phage particles were precipitated from the supernatant by adding $\mathrm{PEG} / \mathrm{NaCl}$ (1/5 volume of the supernatant) and incubated on ice for at least $2 \mathrm{~h}$. Precipitated phage particles were collected by centrifugation (10,000 x $g, 25 \mathrm{~min}$, $4^{\circ} \mathrm{C}$ ), after which the phage pellet was resuspended in $1 \mathrm{~mL}$ of $2 \%$ sarkosyl (Sigma) in phosphate buffered saline (PBS) to remove any unspecific glycan remnants on phage particles and reprecipitated twice with $\mathrm{PEG} / \mathrm{NaCl}$ to wash off the detergent. The phage pellet 
was resuspended in PBS based on pellet size and the phage concentration was determined by $\mathrm{OD}_{268}$ [41]. Phage particles were stored for up to one week at $4^{\circ} \mathrm{C}$ or up to 4 months at $-20^{\circ} \mathrm{C}$.

\section{Western blotting and glycophage ELISA}

For Western blot analysis, glycophages $\left(\sim 6 \times 10^{10}\right.$ phage particles) were run on $7.5 \%$ SDSpolyacrylamide gels (BioRad), and Western blotting was performed as described previously [42]. For glycophage enzyme-linked immunosorbent analysis (ELISA), high-binding 96well clear half-area microtiter plates (Corning) were coated with $1-20 \times 10^{11}$ phage particles in $25 \mu \mathrm{L}$ of PBS buffer in replicates, and incubated for $18-24 \mathrm{~h}$ at $4^{\circ} \mathrm{C}$. The plate was blocked with blocking buffer (PBS $+2 \%$ nonfat milk) for $2 \mathrm{~h}$ at RT. The wells were washed 4 times with PBST (PBS with $0.1 \%$ Tween 20 ) and probed with $50 \mu \mathrm{L}$ of primary antibody and shaken for $1 \mathrm{~h}$ at RT, and if secondary antibody was required, the washed wells were probed for 1 hour at RT. After additional washings with PBS, $o$-phenylenediamine dihydrochloride (OPD) substrate (Sigma) was added to each well and after $40 \mathrm{~min}$ the reaction was stopped with $3 \mathrm{M} \mathrm{H}_{2} \mathrm{SO}_{4}$. The absorbance in each well was measured at 492 $\mathrm{nm}\left(\mathrm{Abs}_{492}\right)$. Blots or arrays were probed with one of the following: anti-MBP antibody conjugated with horseradish peroxidase (HRP) (New England Biolabs), hR6 rabbit serum that is specific for the $C$. jejuni heptasaccharide glycan and also recognizes the $C$. lari hexasaccharide glycan (kindly provided by Markus Aebi), monoclonal antibody 100-4G11A that recognizes the $\mathrm{Man}_{3} \mathrm{GlcNAc}_{2} N$-glycan [43], rabbit anti-E. coli $\mathrm{O} 78$ antibody (Abcam), rabbit anti-E. coli O148 antibody (Abcam), mouse anti-F. tularensis LPS antibody (Abcam), rabbit anti-P. aeruginosa $\mathrm{O} 11$ antibody (kindly provided by Markus Aebi), and Shigella Antiserum Poly Group A raised in rabbit (BD Diagnostic Systems). For non-HRP conjugated antibodies, anti-rabbit IgG-HRP or anti-mouse IgG-HRP (Promega) was used as the secondary antibody. HRP activity was detected using a chemiluminescent substrate (BioRad).

\section{Microarray fabrication}

All chemicals were purchased from Sigma-Aldrich and used without further purification unless otherwise indicated. Non-contact printing was performed on $N$-hydroxysuccinimide (NHS)-activated slides (NEXTERION® Slide H, Schott North America) as previously described [16] as 16 subarrays per slide using a Perkin Elmer Piezorray Printer. For multipanel experiments on a single slide, the array layout was designed using Piezorray software according to the dimension of a standard 16-chamber adaptor. For glycan printing, $100 \mu \mathrm{M}$ of 2-amino- $N$-(2-aminoethyl)-benzamide (AEAB)-conjugated glycans, prepared as previously described [16], were used. All glycan-AEAB (GAEAB) structures are given in Supplementary Fig. S1. For glycophage printing, $10 \mu \mathrm{l}$ of glycophage samples (containing $\sim 1 \times 10^{12} \mathrm{CFU} / \mathrm{mL}$ ) were used. All phage-displayed glycan structures are given in Table 1 . All samples were printed in phosphate buffer ( $100 \mathrm{mM}$ sodium phosphate, $\mathrm{pH} 8.5)$ in replicates of 5, and the average volume delivered to the array was 0.3 nanoliter $+/-10 \%$ (intra-tip). After printing, slides were placed in a high-moisture chamber at $55^{\circ} \mathrm{C}$. After $1 \mathrm{~h}$, the slides were blocked with $50 \mathrm{mM}$ ethanolamine in $0.1 \mathrm{M}$ Tris buffer $(\mathrm{pH} \mathrm{9.0)}$ for $1 \mathrm{~h}$, washed, and subsequently dried by centrifugation and stored desiccated at $-20^{\circ} \mathrm{C}$. For analysis, the 16-chamber adaptor was applied on the slide to separate the single slide to 16 
chambers sealed from each other during the assay. Prior to binding assays, the subarrays were rehydrated for $5 \mathrm{~min}$ in TSM buffer $(20 \mathrm{mM}$ Tris- HCL, $150 \mathrm{mM}$ sodium chloride $(\mathrm{NaCl}), 0.2 \mathrm{mM}$ calcium chloride $\left(\mathrm{CaCl}_{2}\right)$ and $0.2 \mathrm{mM}$ magnesium chloride $\left(\mathrm{MgCl}_{2}\right)$.

\section{GBP binding assays using microarrays}

For interrogation of the printed subarrays, GBPs were diluted in binding buffer (TSM buffer with $1 \%$ BSA and $0.05 \%$ Tween 20 ). The array was interrogated with the following biotinylated lectins (Vector Labs): Helix pomatia agglutinin (HPA), Maakia amurensis lectin (MAL), Sambucus nigra agglutinin (SNA), and soybean agglutinin (SBA), which are specific for defined GAEAB or glycophage structures on the array. An aliquot (200 $\mu$ l) of each GBP or antibody was applied to individual subarrays and incubated for $1 \mathrm{~h}$ at room temperature. After washing with TSM buffer to remove excess GBP, biotinylated lectins were detected by a second incubation with $0.5 \mu \mathrm{g} / \mathrm{ml} \mathrm{Cy5-streptavidin} \mathrm{(Cy5-SA;} \mathrm{Invitrogen}$ Life Technologies), anti-glycan antibodies were detected by a secondary incubation with 5 $\mu \mathrm{g} / \mathrm{ml} \mathrm{Cy5-conjugated} \mathrm{secondary} \mathrm{antibodies} \mathrm{(Invitrogen} \mathrm{Life} \mathrm{Technologies)} \mathrm{at} \mathrm{room}$ temperature for $1 \mathrm{~h}$ followed by a wash step to remove excess reagent and finally with water to remove residual salt.

\section{Microarray scanning}

The slides were scanned with a Perkin Elmer ProScanArray microarray scanner equipped with 4 lasers covering an excitation range from $488 \mathrm{~nm}$ to $637 \mathrm{~nm}$. For Cy5 fluorescence, the excitation wavelength was $649 \mathrm{~nm}$ and the emission wavelength was $670 \mathrm{~nm}$. The scanned images were analyzed with the ScanArray Express software. The results are reported as the average $(n=6)$ relative fluorescence units $(R F U)$ with the error reported as the standard deviation of the mean.

\section{Results}

\section{Redesign of glycophage display system for array fabrication}

We hypothesized that glycan arrays could be fabricated by patterning E. coli-derived glycophages on a solid support such as a nitrocellulose membrane, a microtiter plate, or a glass microscope slide (Fig. 1). Towards this goal, we previously developed a method for the display of asparagine-linked ( $N$-linked) glycans on filamentous phage M13 [17]. At the heart of this system is a plasmid for expressing a fusion between a target $N$-linked glycoprotein and the minor phage coat protein $\mathrm{g} 3 \mathrm{p}$. The target glycoprotein in this plasmid is E. coli $\mathrm{MBP}$, which is modified with a C-terminal glycosylation tag comprising a $\mathrm{D} / \mathrm{E}-\mathrm{X}_{1^{-}}$ $\mathrm{N}-\mathrm{X}_{2}-\mathrm{S} / \mathrm{T}$ acceptor motif that is known to be efficiently glycosylated by the $C$. jejuni oligosaccharyltransferase (OST) PglB [32, 34]. When this plasmid was transformed in $E$. coli cells that also carried the pACYCpgl plasmid encoding the protein glycosylation locus

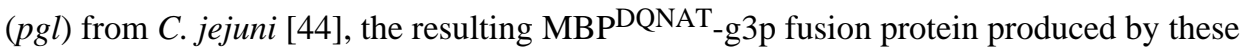
cells was efficiently glycosylated with the $C$. jejuni heptasaccharide glycan ( GalNAc $_{5}(\mathrm{Glc}) \mathrm{Bac}$ where Bac is bacillosamine or 2,4-diacetamido-2,4,6-trideoxyglucose) [17]. Moreover, phage particles prepared from these cells displayed the $C$. jejuni glycan, which was readily detected by glycan-specific serum antibodies or captured by the lectin 
SBA that binds the terminal $N$-acetyl-galactosamine (GalNAc) of the $C$. jejuni oligosaccharide [17].

In this study, the above glycophage system was redesigned for use in glycan array fabrication in several ways. First, we introduced additional acceptor sites for glycan attachment to MBP. This involved replacing $\mathrm{MBP}^{\mathrm{DQNAT}}$ in $\mathrm{pBAD}-\mathrm{MBP}^{\mathrm{DQNAT}}$-g3p with a version of MBP that was C-terminally modified with four tandem repeats of the bacterial glycan acceptor motif DQNAT, resulting in phagemid pBAD-MBP ${ }^{4{ }^{2} D Q N A T}-\mathrm{g} 3 \mathrm{p}$. Second, given the observation that the WaaL O-antigen ligase in E. coli transfers lipid-linked glycans onto lipid A and reduces protein glycosylation efficiency by depleting the pool of available substrates for PglB [45], we used P1vir phage transduction to knockout the waaL gene in $E$. coli strain TG1. Third, we generated the bicistronic phagemid $\mathrm{pBAD}-\mathrm{MBP}^{4 \mathrm{xDQNAT}}$ $\mathrm{g} 3 \mathrm{p}:$ :PglB that encodes both the MBP-g3p fusion and the $C$. jejuni PglB enzyme, thereby affording inducible control over OST expression. Moreover, PglB is needed for transferring other recombinantly produced glycans (e.g., O-antigen polysaccharides) [45]; hence, this bicistronic phagemid provides a copy of the PglB enzyme for future glycan library building. Fourth, because display efficiency and fusion stability often decrease with protein size, possibly as a consequence of coat protein proteolysis, we decreased the overall size of the displayed fusion protein by truncating $\mathrm{g} 3 \mathrm{p}$. This strategy was motivated by the fact that fusion to the C-terminal domain of $\mathrm{g} 3 \mathrm{p}$ can be more stable than fusion to full-length $\mathrm{g} 3 \mathrm{p}$ [46] and also that shortened $\mathrm{g} 3 \mathrm{p}$ enables better display of certain structures by relieving spatial constraints that occur with full-length g3p [47]. Importantly, following helper phage superinfection, the resulting packaged phagemid carries both native g3p of the helper phage, which is necessary for infection, and the encoded fusion comprised of the C-terminal domain of $\mathrm{g} 3 \mathrm{p}$, which is displayed for screening [48].

To determine the overall effectiveness of these modifications, E. coli TG1 $\Delta w a a L$ cells were co-transformed with plasmid pMW07pgl $\Delta \mathrm{B}$ [35] that encodes the entire $C$. jejuni pgl pathway except for PglB and a bicistronic phagemid which encodes PglB and the glycoprotein $\mathrm{MBP}^{4 \times D Q N A T}$ fused to the N-terminus of either full-length $\mathrm{g} 3 \mathrm{p}$ (406 amino acids) or truncated g3p (residues 230-406) [33]. Phage production by these cells was initiated by superinfecting cultures with VCSM13 helper phage followed by induction of fusion protein expression. At the end of the induction phase, the culture densities were virtually identical (data not shown), indicating that the shortened g3p did not adversely affect growth of the host strain. Next, recombinant phage particles were recovered from these cells. The resulting phage titers from TG1 $\Delta$ waaL cells expressing $\mathrm{MBP}^{4 \times D Q N A T}{ }_{-} \mathrm{g} 3 \mathrm{p}$

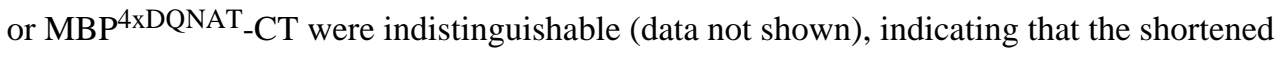
g3p did not adversely affect phage production.

Immunoblot analysis of phage preparations confirmed that the MBP ${ }^{4 D Q N A T}-g 3 p$ and $\mathrm{MBP}^{4 \times D Q N A T}$-CT fusions were incorporated into phage particles at nearly identical levels (Fig. 2A). If the VCSM13 helper phage was omitted, no bands were observed indicating the lack of phage production. In addition, both phage-displayed fusions were glycosylated with the $C$. jejuni glycan as determined by immunoblotting with hR6 serum (Fig. 2A), which is specific for the $C$. jejuni heptasaccharide glycan [36]. When the acceptor motifs were all changed to AQNAT, a sequence that is inefficiently recognized by C. jejuni PglB [34, 35], 
no bands were observed with the hR6 serum indicating that neither of the fusions was glycosylated (Fig. 2A). It is noteworthy that several prominent glycosylated degradation products were observed for the larger $\mathrm{MBP}^{4 \times D Q N A T}-\mathrm{g} 3 \mathrm{p}$ fusion but not the MBP ${ }^{4 \times D Q N A T}$ CT fusion (Fig. 2A), suggesting that the fusion with shortened g3p was more stable under the conditions tested here. For these reasons, we opted to use the pBAD-MBP ${ }^{4 D Q N A T}$. $\mathrm{CT}:$ PglB phagemid for all glycan display hereafter. Using this phagemid under optimal growth and protein expression conditions, we regularly achieved phage titers of $\sim 1 \times 10^{11}$ CFUs per $\mathrm{mL}$ of culture supernatant (Table 1), indicating that very high phage titers could be obtained with our redesigned glycophage system. Importantly, glycosylated phage production using shortened $\mathrm{g} 3 \mathrm{p}$ was found to be dependent on helper phage addition, the phagemid encoding $\mathrm{MBP}^{4 \times D Q N A T}-\mathrm{CT}$, and the glycan biosynthesis pathway

(Supplementary Fig. S2).

\section{Phage-displayed glycan library construction}

Next, we sought to create an array of diverse phage-displayed glycan structures using our redesigned glycophage system. Our strategy was to transform TG1 $\Delta$ waaL cells carrying the pBAD-MBP ${ }^{4 D Q N A T}-C T$ phagemid with a second plasmid encoding a glycan biosynthesis pathway (Table 1). For example, the plasmids pACYCpgl4 and pYCG enabled glycosylation of $\mathrm{MBP}^{4 \times D Q N A T} \mathrm{CT}$ with the $C$. lari hexasaccharide $N$-glycan [36] and the eukaryotic $\mathrm{Man}_{3} \mathrm{GlcNAc}_{2} \mathrm{~N}$-glycan [37], respectively (data not shown). In addition to $\mathrm{N}$ glycans, we also generated phages displaying O-antigen polysaccharides from different Gram-negative bacteria. This was accomplished by leveraging the fact that recombinant Oantigens can be assembled on a lipid carrier (bactoprenyl pyrophosphate) at the cytoplasmic side of the inner membrane of $E$. coli, flipped into the periplasm by the $\mathrm{O}$-antigen translocating flippase Wzx, and transferred to asparagine residues of target proteins by PglB [45]. PglB is the only protein of the bacterial $N$-glycosylation machinery required for this transfer and its relaxed specificity toward the glycan structure enables transfer of numerous structurally distinct O-antigens [38, 45, 49]. To extend this concept to glycophage display, TG1 $\Delta$ waaL cells carrying pBAD-MBP ${ }^{4 \times D Q N A T}$-CT were transformed with plasmid pMW07-O78, which encodes enzymes for the biosynthesis of Ec-OAg (O78) composed of tetrasaccharide repeating-units whose structure is given in Table 1. High recombinant phage titers were recovered from these cells, similar to the titers achieved with the different $\mathrm{N}$ glycan biosynthesis pathways (Table 1). When these phage preparations were analyzed by immunoblotting, a ladder of higher molecular weight bands were detected by the anti-EcOAg (O78) antiserum (Fig. 2B). This laddering of O78 polysaccharide-linked phages was consistent with the typical variability of the chain length of the O-polysaccharides generated by the Wzy polymerase [45]. The high-molecular-weight bands were absent in the samples obtained from cells that were not infected with helper phage, lacked the phagemid, or lacked the O-antigen biosynthesis pathway (Fig. 2B). Altogether, these results suggest that $\mathrm{O} 78$ polysaccharide was covalently linked to $\mathrm{MBP}^{4 \mathrm{XDQNAT}}$-CT. Nearly identical phage glycosylation was obtained for four additional O-antigens including: Ec-OAg (O148) produced from pMW07-O148; Ft-OAg produced from pGAB2 [38]; Pa-OAg produced from pLPS2 [39]; and Sd-OAg produced from pSS37 [40]. The structures of these antigens and their production levels are summarized in Table 1. 


\section{Detection of glycan-protein interactions by glycophage ELISA}

We next determined whether glycans displayed on the surface of phages could still be detected by GBPs following immobilization to a solid surface. Various methods have been employed to immobilize phages on surfaces for array development including noncovalent adsorption to polystyrene or nitrocellulose [28, 29]. Indeed, when glycophages prepared from TG1 $\Delta$ waaL cells carrying the pBAD-MBP ${ }^{4 x_{D Q N A T}}$ CT::PglB phagemid and plasmid $\mathrm{pMW} 07 \mathrm{pgl} \triangle \mathrm{B}$ were used to coat the surface of microtiter plates, a strong binding signal was observed following incubation with hR6 serum antibodies (Fig. 3A). In contrast, only background signals were measured for the helper phage control or the glycophage sample prepared from TG1 $\Delta$ waaL cells carrying pMW07pgl $\Delta \mathrm{B}$ along with the pBADMBP ${ }^{4 \times A Q N A T}$-CT::PglB phagemid, in which the acceptor motifs have been mutated (Fig. 3A). As expected, ELISA signals for all of the other immobilized glycophages were significantly lower than that of the $C$. jejuni $N$-glycan, except for the $C$. lari hexasaccharide glycan which is structurally similar to the $C$. jejuni $N$-glycan and reacts with hR6 serum [36]. Collectively, these data indicate that glycophages are compatible with surface immobilization procedures and that phage-displayed glycans retain the ability to be recognized by a cognate GBP after immobilization. Identical glyophage ELISAs were performed for six additional GBPs that recognized one of the glycans in the library. The resulting dataset was used to generate an orthogonality matrix of glycan binding for each of the GBPs (Fig. 3B). Importantly, all of the GBPs bound most efficiently to their target glycan structures as evidenced by the strong blue signal along the diagonal of the matrix. The signal-to-noise ratios (S:N) generated using this assay were significant, with values of 3.0, 3.5, 6.7, and 10 for the anti-Cj hepta, anti-Cl hexa, anti-FtOAg, anti-PaOAg antibodies, respectively (where $\mathrm{S}: \mathrm{N}$ was determined by normalizing the activity measured for GBP binding of cognate glycan (signal) by the highest activity measured for GBP binding of noncognate glycan (noise). For the poorer S:N cases, such as the anti-Ec-OAg antibodies ( $\mathrm{S}: \mathrm{N}=$ 1.9 for $\mathrm{O} 78$ and 1.8 for O148) and the anti-SD-OAg antibody ( $\mathrm{S}: \mathrm{N}=1.5)$, it is unclear whether this cross reactivity was due to unexpectedly low specificity of the GBPs themselves or the need for further optimization of assay conditions to reduce background caused by non-specific cross-reaction (e.g., reduce possible phage cross-contamination which arises more readily than bacterial contaminations, but can be prevented using filtered tips).

\section{Fabrication of glycophage-patterned microarrays}

Encouraged by these ELISA results, we sought to prepare glycan arrays by directly spotting recombinant glycophages onto the surface of glass slides. Previous studies have shown that phages displaying peptides and proteins are compatible with robotic microarray spotters [26-28] and covalent chemical conjugation to glass slides through amino acids (e.g., lysine, cysteine, or any $\mathrm{N}$ - or C-terminal acids) that provide a suitable functional capacity for immobilization and modification chemistries [26]. Here, a similar strategy was adopted for covalently attaching glycophages to microglass slides by using standard microarray printing technology $[16,50]$. Specifically, we used commercially available amine-reactive NHSactivated glass slides, which allowed rapid covalent coupling of terminal amines in glycophage coat proteins via formation of an amide bond under aqueous conditions at room 
temperature. In addition to spotting glycophage samples, we also printed PBS, biotin, and 10 different amine-functionalized GAEABs from the repository of the Consortium for Functional Glycomics (CFG) [16] (Supplementary Fig. S1), which served as controls for array fabrication and GBP binding.

To validate glycophage printing, we probed our microarrays with GBPs having specificity for the different glycans that were printed on the slides. For example, probing with serum antibodies against the $P$. aeruginosa $\mathrm{O} 11 \mathrm{O}$-antigen followed by a Cy5-labeled secondary antibody resulted in strong binding to glycophages displaying the $\mathrm{O} 11 \mathrm{O}$-antigen but not to other glycophages or any of the GAEAB control glycans (Fig. 4A and Supplementary Figs. S3 and S4). When the same array was probed with biotinylated lectins, HPA, MAL, and SNA and detected using Cy5-SA [16], only binding to cognate GAEABs was observed (Fig. 4A and Supplementary Fig. S3 and S4), consistent with their reported specificities. Importantly, none of these lectins bound to any of the glycophages. Nearly identical results were obtained with a second array that varied only in the identity and number of some of the GAEABs and glycophages that were printed. In particular, we observed that biotinylated SBA, which recognizes terminal GalNAc residues such as those in the $C$. jejuni and $C$. lari $\mathrm{N}$-glycans [17], bound strongly and selectively to glycophages displaying the $C$. jejuni $N$ glycan structure (Fig. 4B). Collectively, these results signify that glycophages can be printed on NHS-derivatized microglass slides using standard robotic printing technology and that covalently linked phages retain their ability to be recognized by GBPs.

\section{Discussion}

Glycan arrays have emerged in the last decade as a powerful tool for analyzing carbohydrate-macromolecule interactions, especially in disease related research. Microarrays are specifically preferred because they facilitate rapid screening of numerous samples and substantially reduce reagent consumption. However, unlike their DNA and protein counterparts, glycans cannot be readily amplified in vitro. Hence, acquiring a large collection of glycans in a format suitable for immobilization on the array surface remains a formidable challenge given the low amounts of glycan that can be chemically synthesized or isolated from natural sources. In this study, we demonstrate an alternative approach to glycan arrays that leverages the biosynthetic potential of engineered $E$. coli to covalently link tailor-made $\mathrm{N}$-glycans and $\mathrm{O}$-antigens to filamentous phage particles in vivo. The resulting glycan-displaying phages accumulate outside the cells and thus can be easily recovered from the culture media without the need for cell lysis or laborious purification techniques. In fact, the phage recovery method is completely independent of the glycan structure, which effectively streamlines the entire glycan library production process. In addition to facile production, the method also yields large quantities of glycans with even greater quantities of glycans possible in the future given the scalability of bacterial cell culture. Most importantly, we show that glycophages are entirely compatible with every step of the microarray fabrication process, and can be immobilized to a support surface by either noncovalent or covalent chemistries in a manner that does not disrupt their ability to be recognized by different GBPs (e.g., antibodies, lectins) after immobilization. 
Using both microtiter plates and glass slides, significant $\mathrm{S}: \mathrm{N}$ ratios were obtained for a panel of GBP-glycan pairs; however, a fair amount of unexpected or non-specific glycan binding was observed with the microtiter plate format. This background was not seen for the limited set of GBP-glycan pairs tested using the glass slide arrays, suggesting this may be the preferred format for future glycomic applications. An issue that remains unanswered is the density of glycans on the different glycophages, which is not currently known and is difficult to accurately quantify due to the relatively low level of phage glycosylation [17, 18]. For a different glycoprotein target, namely $C$. jejuni AcrA, the efficiency with which different recombinant O-antigens were conjugated by $C$. jejuni PglB was variable [45]. This is significant because GBPs typically bind to clustered glycans, and if the glycan densities vary significantly among the glycophages, conclusions about binding specificity will be compromised. Hence, future efforts must focus on techniques for quantifying glycan density and controlling/modulating the glycosylation efficiency of PglB. Another limitation of the technology at present is the fact that de novo biosynthesis of diverse glycoforms (i.e., candidate glycan library members) in engineered bacteria is in its infancy (for recent reviews on the topic, see [51, 52]). The silver lining, however, is that every newly engineered glycoform produced using $E$. coli in the future will potentially serve as a library member for glycophage array development. In addition, it may be possible to diversify the panel of existing glycan structures by combining in vivo glycophage production with in vitro remodeling of phage-displayed glycans using chemoenzymatic approaches as described recently [53]. In light of these possibilities, our results pave the way for future robotic printing of large panels of glycan epitopes displayed on phages for highly parallel, highthroughput profiling of GBP specificities in experimental and clinical samples.

\title{
Supplementary Material
}

Refer to Web version on PubMed Central for supplementary material.

\section{Acknowledgements}

\begin{abstract}
We thank Brendan Wren, Markus Aebi, Rebecca Thomas, Renato Morona, and Chung-Jr Huang for plasmids and antiserum used in this work. We also thank Julius Lucks for help preparing figures. The National Center for Functional Glycomics is supported by NIGMS grant P41 GM103694. This work was also supported by the National Science Foundation Career Award CBET-0449080 (to M.P.D.), the New York State Office of Science, Technology and Academic Research Distinguished Faculty Award (to M.P.D.), National Institutes of Health grant \# 1 R43 GM086965-01 (to A.C.F), and by a Marie Curie FP7 Integration Grant within the 7th European Union Framework Programme under REA grant agreement \# 322096 (to E.C.), UNESCO-L'Oréal National Young Women in Science Award (to E.C.), and Hacettepe University (to E.C.).
\end{abstract}

\section{Abbreviations}

$\begin{array}{ll}\text { AEAB } & \text { 2-amino- } N \text {-(2-aminoethyl)-benzamide } \\ \text { CFU } & \text { asparagine-linked ( } N \text {-linked), colony-forming unit } \\ \text { CT } & \text { C-terminal domain of g3p } \\ \text { Cy5-SA } & \text { Cy5-streptavidin } \\ \text { ELISA } & \text { enzyme-linked immunosorbent assay }\end{array}$




$\begin{array}{ll}\text { GBP } & \text { glycan-binding protein } \\ \text { GAEAB } & \text { glycan-AEAB } \\ \text { HPA } & \text { Helix pomatia agglutinin } \\ \text { MAL } & \text { Maakia amurensis lectin } \\ \text { MBP } & \text { maltose-binding protein } \\ \text { Man3 } & \text { Man }_{3} \text { GlcNAc }_{2} \\ \text { NHS } & N_{\text {-hydroxysuccinimide }} \\ \text { OST } & \text { oligosaccharyltransferase } \\ \text { SNA } & \text { Sambucus nigra } \text { agglutinin } \\ \text { SBA } & \text { soybean agglutinin } \\ \text { RFU } & \text { relative fluorescence unit }\end{array}$

\section{References}

1. Varki A. Biological roles of oligosaccharides: all of the theories are correct. Glycobiology. 1993; 3:97-130. [PubMed: 8490246]

2. Freeze HH. Genetic defects in the human glycome. Nat Rev Genet. 2006; 7:537-551. [PubMed: 16755287]

3. Rillahan CD, Paulson JC. Glycan microarrays for decoding the glycome. Annu Rev Biochem. 2011; 80:797-823. [PubMed: 21469953]

4. Oyelaran O, Gildersleeve JC. Glycan arrays: recent advances and future challenges. Curr Opin Chem Biol. 2009; 13:406-413. [PubMed: 19625207]

5. Smith DF, Song X, Cummings RD. Use of glycan microarrays to explore specificity of glycanbinding proteins. Methods Enzymol. 2010; 480:417-444. [PubMed: 20816220]

6. de Paz JL, Seeberger PH. Recent advances and future challenges in glycan microarray technology. Methods Mol Biol. 2012; 808:1-12. [PubMed: 22057514]

7. Song X, Heimburg-Molinaro J, Cummings RD, Smith DF. Chemistry of natural glycan microarrays. Curr Opin Chem Biol. 2014; 18:70-77. [PubMed: 24487062]

8. Park S, Gildersleeve JC, Blixt O, Shin I. Carbohydrate microarrays. Chem Soc Rev. 2013; 42:43104326. [PubMed: 23192235]

9. Wang D, Liu S, Trummer BJ, Deng C, Wang A. Carbohydrate microarrays for the recognition of cross-reactive molecular markers of microbes and host cells. Nat Biotechnol. 2002; 20:275-281. [PubMed: 11875429]

10. Arthur CM, Cummings RD, Stowell SR. Using glycan microarrays to understand immunity. Curr Opin Chem Biol. 2014; 18:55-61. [PubMed: 24486647]

11. Stowell SR, Arthur CM, McBride R, Berger O, et al. Microbial glycan microarrays define key features of host-microbial interactions. Nat Chem Biol. 2014; 10:470-476. [PubMed: 24814672]

12. Adams EW, Ratner DM, Bokesch HR, McMahon JB, et al. Oligosaccharide and glycoprotein microarrays as tools in HIV glycobiology; glycan-dependent gp120/protein interactions. Chem Biol. 2004; 11:875-881. [PubMed: 15217620]

13. Lawrie CH, Marafioti T, Hatton CS, Dirnhofer S, et al. Cancer-associated carbohydrate identification in Hodgkin's lymphoma by carbohydrate array profiling. Int J Cancer. 2006; 118:3161-3166. [PubMed: 16395706]

14. Oyelaran O, Gildersleeve JC. Application of carbohydrate array technology to antigen discovery and vaccine development. Expert Rev Vaccines. 2007; 6:957-969. [PubMed: 18377358] 
15. Wang D, Carroll GT, Turro NJ, Koberstein JT, et al. Photogenerated glycan arrays identify immunogenic sugar moieties of Bacillus anthracis exosporium. Proteomics. 2007; 7:180-184. [PubMed: 17205603]

16. Song X, Xia B, Stowell SR, Lasanajak Y, et al. Novel fluorescent glycan microarray strategy reveals ligands for galectins. Chem Biol. 2009; 16:36-47. [PubMed: 19171304]

17. Celik E, Fisher AC, Guarino C, Mansell TJ, DeLisa MP. A filamentous phage display system for N-linked glycoproteins. Protein Sci. 2010; 19:2006-2013. [PubMed: 20669235]

18. Durr C, Nothaft H, Lizak C, Glockshuber R, Aebi M. The Escherichia coli glycophage display system. Glycobiology. 2010; 20:1366-1372. [PubMed: 20581006]

19. Smith GP. Filamentous fusion phage: novel expression vectors that display cloned antigens on the virion surface. Science. 1985; 228:1315-1317. [PubMed: 4001944]

20. Winter G, Griffiths AD, Hawkins RE, Hoogenboom HR. Making antibodies by phage display technology. Annu Rev Immunol. 1994; 12:433-455. [PubMed: 8011287]

21. Rader C, Barbas CF 3rd. Phage display of combinatorial antibody libraries. Curr Opin Biotechnol. 1997; 8:503-508. [PubMed: 9265732]

22. Smith GP. Surface presentation of protein epitopes using bacteriophage expression systems. Curr Opin Biotechnol. 1991; 2:668-673. [PubMed: 1370065]

23. Viti F, Nilsson F, Demartis S, Huber A, Neri D. Design and use of phage display libraries for the selection of antibodies and enzymes. Methods Enzymol. 2000; 326:480-505. [PubMed: 11036659]

24. Watters AL, Baker D. Searching for folded proteins in vitro and in silico. Eur J Biochem. 2004; 271:1615-1622. [PubMed: 15096200]

25. Forrer P, Jung S, Pluckthun A. Beyond binding: using phage display to select for structure, folding and enzymatic activity in proteins. Curr Opin Struct Biol. 1999; 9:514-520. [PubMed: 10449374]

26. Bi Q, Cen X, Wang W, Zhao X, et al. A protein microarray prepared with phage-displayed antibody clones. Biosens Bioelectron. 2007; 22:3278-3282. [PubMed: 17418563]

27. Cekaite L, Haug O, Myklebost O, Aldrin M, et al. Analysis of the humoral immune response to immunoselected phage-displayed peptides by a microarray-based method. Proteomics. 2004; 4:2572-2582. [PubMed: 15352232]

28. Arnaud MC, Gazarian T, Palacios Rodriguez Y, Gazarian K, Sakanyan V. Array assessment of phage-displayed peptide mimics of Human Immunodeficiency Virus type 1 gp41 immunodominant epitope: binding to antibodies of infected individuals. Proteomics. 2004; 4:1959-1964. [PubMed: 15221756]

29. Zhong L, Hidalgo GE, Stromberg AJ, Khattar NH, et al. Using protein microarray as a diagnostic assay for non-small cell lung cancer. Am J Respir Crit Care Med. 2005; 172:1308-1314. [PubMed: 16109979]

30. Baba T, Ara T, Hasegawa M, Takai Y, et al. Construction of Escherichia coli K-12 in-frame, single-gene knockout mutants: the Keio collection. Mol Syst Biol. 2006; 2:0008. 2006. [PubMed: 16738554]

31. Hertveldt K, Belien T, Volckaert G. General M13 phage display: M13 phage display in identification and characterization of protein-protein interactions. Methods Mol Biol. 2009; 502:321-339. [PubMed: 19082565]

32. Fisher AC, Haitjema CH, Guarino C, Celik E, et al. Production of secretory and extracellular Nlinked glycoproteins in Escherichia coli. Appl Environ Microbiol. 2011; 77:871-881. [PubMed: 21131519]

33. Andris-Widhopf J, Rader C, Steinberger P, Fuller R, Barbas CF 3rd. Methods for the generation of chicken monoclonal antibody fragments by phage display. J Immunol Methods. 2000; 242:159_ 181. [PubMed: 10986398]

34. Kowarik M, Young NM, Numao S, Schulz BL, et al. Definition of the bacterial N-glycosylation site consensus sequence. EMBO J. 2006; 25:1957-1966. [PubMed: 16619027]

35. Ollis AA, Fisher AC, Merritt JH, Zhang S, DeLisa MP. Engineered oligosaccharyltransferases with greatly relaxed acceptor site specificity. Nat Chem Biol. 2014 (in press).

36. Schwarz F, Lizak C, Fan YY, Fleurkens S, et al. Relaxed acceptor site specificity of bacterial oligosaccharyltransferase in vivo. Glycobiology. 2011; 21:45-54. [PubMed: 20847188] 
37. Valderrama-Rincon JD, Fisher AC, Merritt JH, Fan YY, et al. An engineered eukaryotic protein glycosylation pathway in Escherichia coli. Nat Chem Biol. 2012; 8:434-436. [PubMed: 22446837]

38. Cuccui J, Thomas RM, Moule MG, D'Elia RV, et al. Exploitation of bacterial N-linked glycosylation to develop a novel recombinant glycoconjugate vaccine against Francisella tularensis. Open Biol. 2013; 3:130002. [PubMed: 23697804]

39. Goldberg JB, Hatano K, Meluleni GS, Pier GB. Cloning and surface expression of Pseudomonas aeruginosa O antigen in Escherichia coli. Proc Natl Acad Sci U S A. 1992; 89:10716-10720. [PubMed: 1279692]

40. Sturm S, Timmis KN. Cloning of the rfb gene region of Shigella dysenteriae 1 and construction of an rfb-rfp gene cassette for the development of lipopolysaccharide-based live anti-dysentery vaccines. Microb Pathog. 1986; 1:289-297. [PubMed: 2469931]

41. Sidhu SS, Lowman HB, Cunningham BC, Wells JA. Phage display for selection of novel binding peptides. Methods Enzymol. 2000; 328:333-363. [PubMed: 11075354]

42. DeLisa MP, Tullman D, Georgiou G. Folding quality control in the export of proteins by the bacterial twin-arginine translocation pathway. Proc Natl Acad Sci U S A. 2003; 100:6115-6120. [PubMed: 12721369]

43. van Remoortere A, Bank CM, Nyame AK, Cummings RD, et al. Schistosoma mansoni-infected mice produce antibodies that cross-react with plant, insect, and mammalian glycoproteins and recognize the truncated biantennaryN-glycan Man3GlcNAc2-R. Glycobiology. 2003; 13:217-225. [PubMed: 12626421]

44. Wacker M, Linton D, Hitchen PG, Nita-Lazar M, et al. N-linked glycosylation in Campylobacter jejuni and its functional transfer into E. coli. Science. 2002; 298:1790-1793. [PubMed: 12459590]

45. Feldman MF, Wacker M, Hernandez M, Hitchen PG, et al. Engineering N-linked protein glycosylation with diverse $\mathrm{O}$ antigen lipopolysaccharide structures in Escherichia coli. Proc Natl Acad Sci U S A. 2005; 102:3016-3021. [PubMed: 15703289]

46. Larocca D, Jensen-Pergakes K, Burg MA, Baird A. Receptor-targeted gene delivery using multivalent phagemid particles. Mol Ther. 2001; 3:476-484. [PubMed: 11319907]

47. Lowman HB, Bass SH, Simpson N, Wells JA. Selecting high-affinity binding proteins by monovalent phage display. Biochemistry. 1991; 30:10832-10838. [PubMed: 1932005]

48. Barbas CF 3rd, Kang AS, Lerner RA, Benkovic SJ. Assembly of combinatorial antibody libraries on phage surfaces: the gene III site. Proc Natl Acad Sci U S A. 1991; 88:7978-7982. [PubMed: 1896445]

49. Ihssen J, Kowarik M, Dilettoso S, Tanner C, et al. Production of glycoprotein vaccines in Escherichia coli. Microb Cell Fact. 2010; 9:61. [PubMed: 20701771]

50. Blixt O, Head S, Mondala T, Scanlan C, et al. Printed covalent glycan array for ligand profiling of diverse glycan binding proteins. Proc Natl Acad Sci U S A. 2004; 101:17033-17038. [PubMed: 15563589]

51. Merritt JH, Ollis AA, Fisher AC, DeLisa MP. Glycans-by-design: engineering bacteria for the biosynthesis of complex glycans and glycoconjugates. Biotechnol Bioeng. 2013; 110:1550-1564. [PubMed: 23456823]

52. Baker JL, Celik E, DeLisa MP. Expanding the glycoengineering toolbox: the rise of bacterial Nlinked protein glycosylation. Trends Biotechnol. 2013; 31:313-323. [PubMed: 23582719]

53. Schwarz F, Huang W, Li C, Schulz BL, et al. A combined method for producing homogeneous glycoproteins with eukaryotic N-glycosylation. Nat Chem Biol. 2010; 6:264-266. [PubMed: 20190762]

54. Young NM, Brisson JR, Kelly J, Watson DC, et al. Structure of the N-linked glycan present on multiple glycoproteins in the Gram-negative bacterium, Campylobacter jejuni. J Biol Chem. 2002; 277:42530-42539. [PubMed: 12186869]

55. Jansson PE, Lindberg B, Widmalm G, Leontein K. Structural studies of the Escherichia coli O78 O-antigen polysaccharide. Carbohydr Res. 1987; 165:87-92. [PubMed: 2444338]

56. Feng L, Perepelov AV, Zhao G, Shevelev SD, et al. Structural and genetic evidence that the Escherichia coli O148 O antigen is the precursor of the Shigella dysenteriae type $1 \mathrm{O}$ antigen and identification of a glucosyltransferase gene. Microbiology. 2007; 153:139-147. [PubMed: 17185542] 
57. Vinogradov EV, Shashkov AS, Knirel YA, Kochetkov NK, et al. Structure of the O-antigen of Francisella tularensis strain 15. Carbohydr Res. 1991; 214:289-297. [PubMed: 1769021]

58. Knirel Yu A, Vinogradov EV, Kocharova NA, Paramonov NA, et al. The structure of O-specific polysaccharides and serological classification of Pseudomonas aeruginosa (a review). Acta Microbiol Hung. 1988; 35:3-24. [PubMed: 3134788]

59. Dmitriev BA, Knirel YA, Kochetkov NK. Somatic antigens of shigella. Structural investigation on the O-specific polysaccharide chain of Shigella dysenteriae type 1 lipopolysaccharide. Eur J Biochem. 1976; 66:559-566. [PubMed: 8314] 


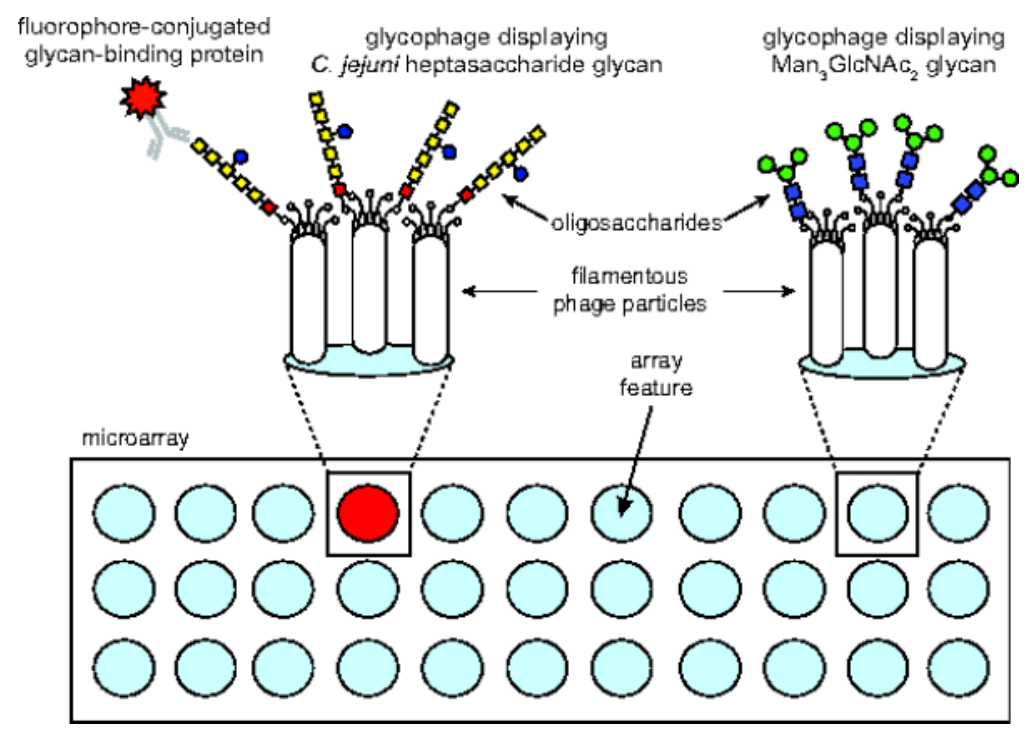

Figure 1. Schematic of glycophage array technology

Detection of GBP binding activity enabled by covalent printing of diverse glycophages, each displaying a different glycan, onto an amino-reactive glass surface followed by image analysis using standard microarray technology. The displayed glycan is produced biosynthetically in engineered E. coli cells carrying a plasmid that encodes the glycan biosynthesis pathway and a phagemid that encodes the acceptor protein-phage coat protein fusion (e.g., MBP $\mathrm{MDQNAT}_{-} \mathrm{g} 3 \mathrm{p}$ ). In the presence of the OST PglB from the bacterium $C$. jejuni, structurally diverse glycans can be covalently linked to $\mathrm{MBP}^{4 \mathrm{xDQNAT}_{-} \mathrm{g} 3 \mathrm{p} \text { and }}$ subsequently displayed on phage particles. 


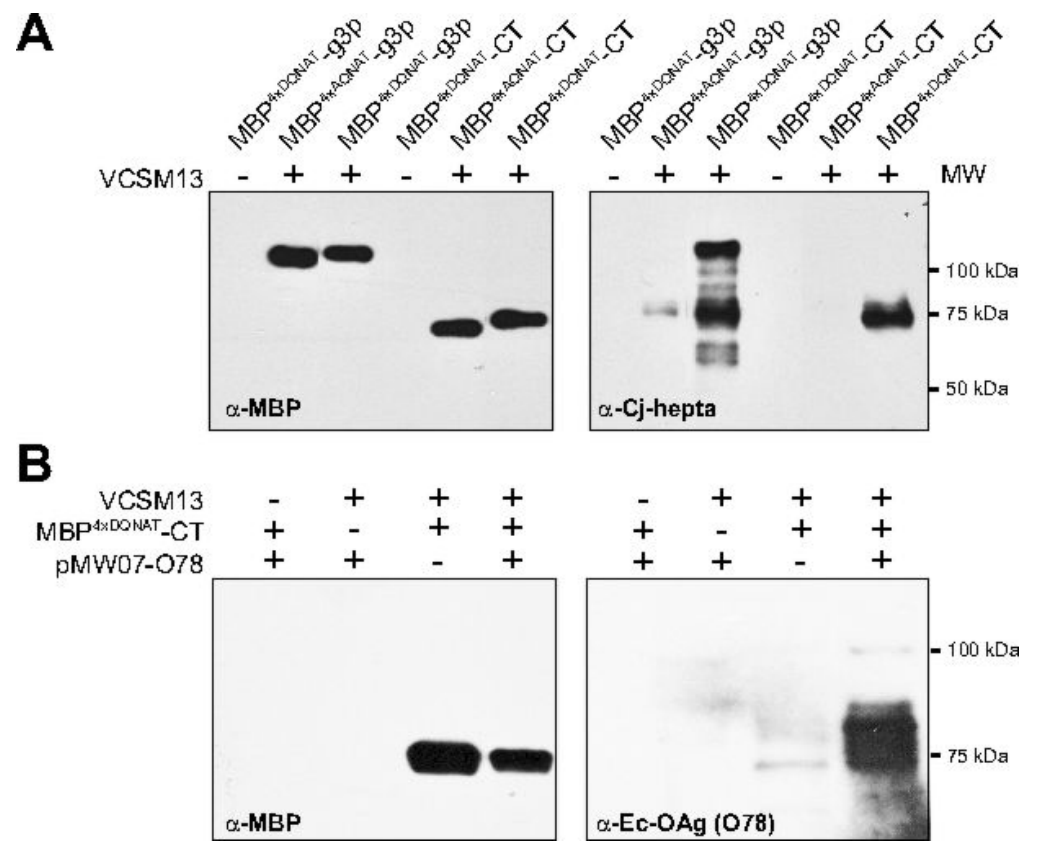

Figure 2. Generation of phage-displayed glycan library members

(A) Western blot analysis of phage preparations (containing $4 \times 10^{10}$ glycophage particles) derived from $E$. coli TG1 $\Delta$ waaL cells co-transformed with pMW07pgl $\Delta \mathrm{B}$ and one of the following phagemids: pBAD-MBP ${ }^{4 D Q N A T}$-g3p::PglB, pBAD-MBP4xAQNAT pBADMBP4xDQNAT_CT::PglB or pBAD-MBP4xAQNAT_CT::PglB. (B) Western blot analysis of phage preparations (containing $6 \times 10^{10}$ glycophage particles) derived from $E$. coli TG1 $\Delta$ waaL cells co-transformed with phagemid pBAD-MBP ${ }^{4 D Q N A T}-\mathrm{CT}:: P g l B$ and pMW07-O78. Phage preparation involved addition (+) or omission (-) of VCSM13 helper phage. Additional controls involved omitting either the phagemid or the glycan biosynthesis pathway. Blots were probed with anti-MBP, hR6 serum (anti-Cj-hepta), or anti-Ec-OAg (O78) as indicated. Molecular weight (MW) markers are indicated at right. 


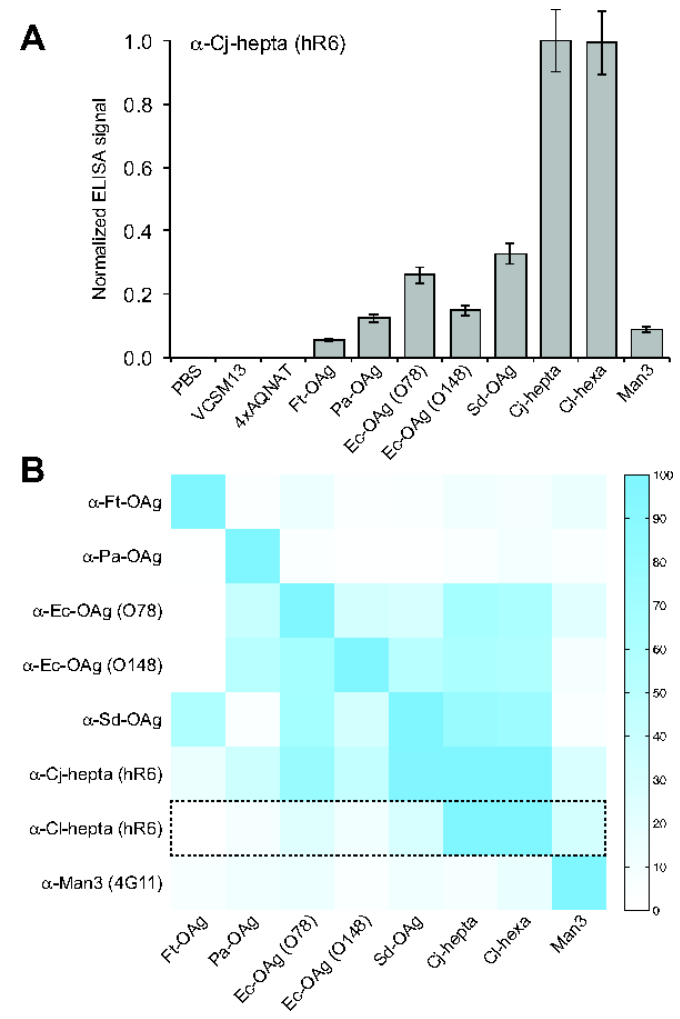

Figure 3. Glycan-protein interactions detected by glycophage ELISA

(A) Representative ELISA results obtained by immobilizing glycophage preparations on microtiter plate surfaces. Glycophage preparations were prepared from E. coli TG1 $\Delta w a a L$

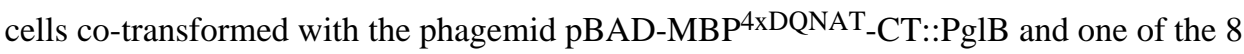
different glycan encoding plasmids. Controls included PBS, helper phage only (VCSM13), and phages prepared from E. coli TG1 $\Delta w a a L$ cells co-transformed with pBAD$\mathrm{MBP}^{4 \times A Q N A T}$-CT::PglB and pMW07pgl $\triangle \mathrm{B}$ (4xAQNAT). All samples were immobilized in triplicate and probed with hR6 serum antibodies (a-Cj-hepta). ELISA signals ( $\mathrm{Abs}_{492}$ ) for each sample were normalized to the signal measured for the Cjhepta $N$-glycan sample. The results are reported as the average normalized ELISA signal $(n=3)$ and error bars represent the standard deviation of the mean. (B) Orthogonality matrix depicting glycan specificity profiles for different GBPs. Glycophage ELISA was performed whereby each GBP (y-axis) was probed against the glycan library (x-axis) identically as described in (A) for hR6 serum antibodies. Each row depicts the normalized ELISA signals measured for the indicated GBP against each glycan sample, where data was independently normalized to the maximum signal obtained for that GBP. Color intensity scale is shown at right (highest signal = blue; lowest signal $=$ white) . Dashed box corresponds to data from $(A)$ for hR6 serum antibodies. 


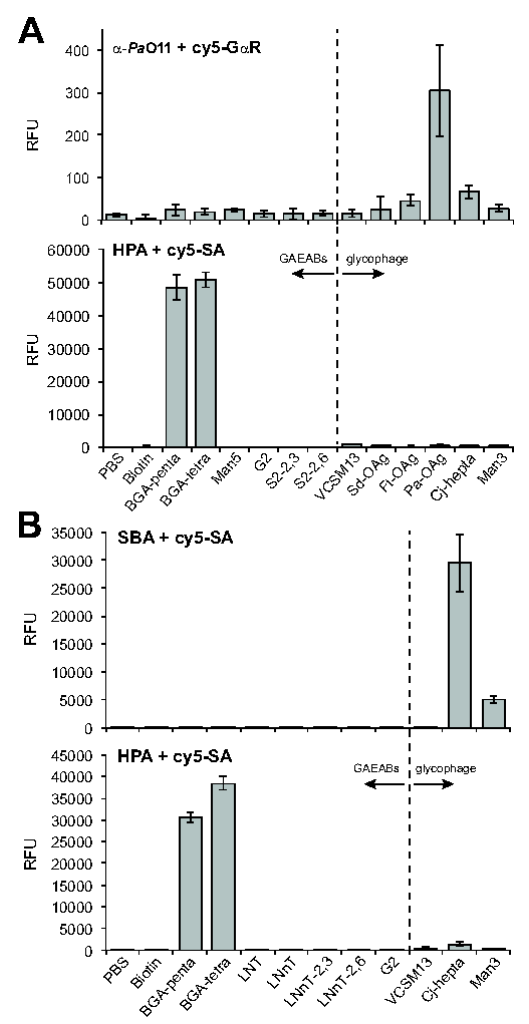

Figure 4. Detection of phage-displayed $\mathrm{O}$-antigens using glycan microarray (A) Glycan binding by serum antibodies against Pa-OAg was detected with Cy5-labeled goat anti-rabbit antibodies (Cy5-GaR) while binding by lectin HPA was detected with Cy5SA. Arrays were printed with the following: PBS, biotin, blood group A pentasaccharide (BGA-penta), BGA tetrasaccharide (BGA-tetra), Man 5 GlcNAc $_{2}$ (Man5), biantennary nonsialylated G2 glycan (G2), biantennary disialylated glycan (a2,3 linkage; S2-2,3), biantennary disialylated glycan (a2,6 linkage; S2-2,6), helper phage control (VCSM13), and phages displaying Sd-OAg, Ft-OAg, Pa-OAg, Cj-hepta and Man3. Data reported as the average $(n=6)$ RFU and error bars represent the standard deviation of the mean. (B) Glycan binding by the lectins SBA and HPA was detected with Cy5-SA. Arrays were printed with the following: PBS, biotin, blood-group A pentasaccharide (BGA-penta), BGA tetrasaccharide (BGA-tetra), lacto- $N$-tetraose (LNT), lacto- $N$-neotetraose (LNnT), sialyllacto- $N$-neotetraose (a2,3 linkage; LNnT-2,3), sialyllacto- $N$-neotetraose (a2,6 linkage, LNnT-2,6), biantennary non-sialylated G2 glycan (G2), helper phage control (VCSM13), and phages displaying $\mathrm{Cj}$-hepta and Man3. Data reported as average $(n=6)$ RFU and error bars represent the standard deviation of the mean. 


\section{Table 1}

Summary of phage-displayed glycan library construction

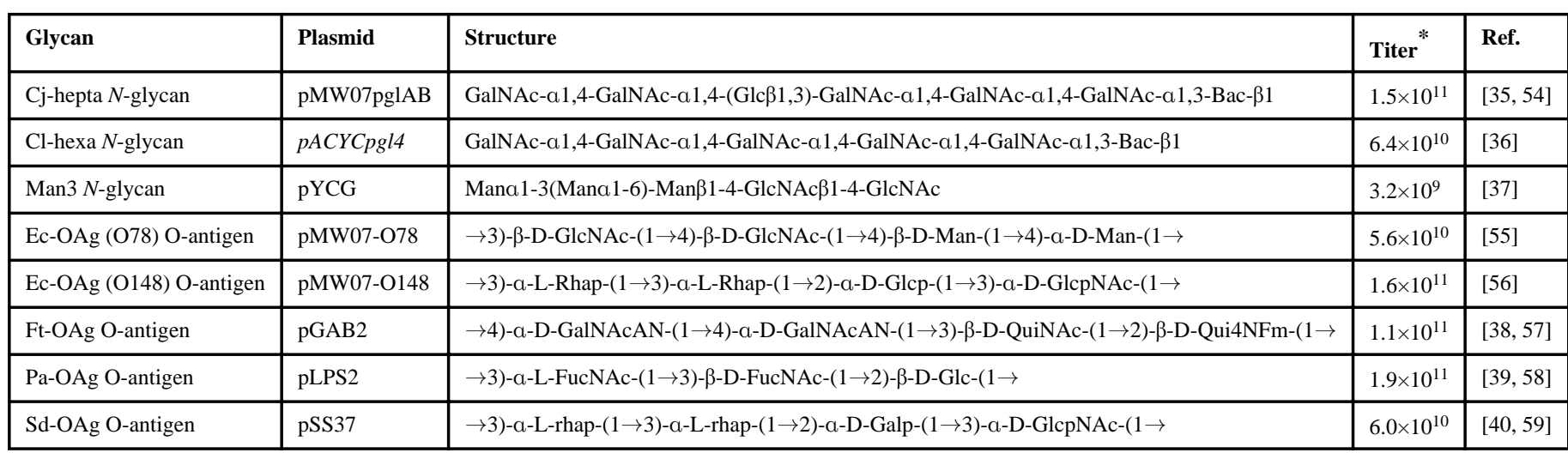

* CFU/mL culture supernatant 